\title{
The Effectiveness of Pedagogical Agents' Prompting and Feedback in Facilitating Co-adapted Learning with MetaTutor
}

\author{
Roger Azevedo ${ }^{1}$, Ronald S. Landis ${ }^{2}$, Reza Feyzi-Behnagh ${ }^{1}$, Melissa Duffy ${ }^{1}$, \\ Gregory Trevors ${ }^{1}$, Jason M. Harley ${ }^{1}$, François Bouchet ${ }^{1}$, Jonathan Burlison ${ }^{3}$, \\ Michelle Taub ${ }^{1}$, Nicole Pacampara ${ }^{1}$, Mohamed Yeasin ${ }^{4}$, A.K.M. Mahbubur Rahman ${ }^{4}$, \\ M. Iftekhar Tanveer ${ }^{4}$, and Gahangir Hossain ${ }^{4}$ \\ ${ }^{1}$ McGill University, Dept. of Educational and Counselling Psychology, Montreal, Canada \\ roger.azevedo@mcgill.ca \\ ${ }^{2}$ Illinois Institute of Technology, College of Psychology, Chicago, IL, USA \\ rlandis@iit.edu \\ ${ }^{3}$ University of Memphis, Dept. of Psychology, Memphis, TN, USA \\ jaburlsnayahoo.com \\ ${ }^{4}$ University of Memphis, Dept. of Electrical and Computer Engineering, Memphis, TN, USA \\ myeasin@memphis.edu
}

\begin{abstract}
Co-adapted learning involves complex, dynamically unfolding interactions between human and artificial pedagogical agents (PAs) during learning with intelligent systems. In general, these interactions lead to effective learning when (1) learners correctly monitor and regulate their cognitive and metacognitive processes in response to internal (e.g., accurate metacognitive judgments followed by the selection of effective learning strategies) and external (e.g., response to agents' prompting and feedback) conditions, and (2) pedagogical agents can adequately and correctly detect, track, model, and foster learners' self-regulatory processes. In this study, we tested the effectiveness of PAs' prompting and feedback on learners' self-regulated learning about the human circulatory system with MetaTutor, an adaptive, multi-agent learning environment. Sixty-nine $(N=69)$ undergraduates learned about the topic with MetaTutor, during a 2-hour session under one of three conditions: prompt and feedback (PF), prompt-only (PO), and no prompt (NP) condition. The PF condition received timely prompts from several pedagogical agents to deploy various SRL processes and received immediate directive feedback concerning the deployment of the processes. The PO condition received the same timely prompts, without feedback. Finally, the NP condition learned without assistance from the agents. Results indicate that those in the PF condition had significantly higher learning efficiency scores than those in both the PO and control conditions. In addition, log-file data provided evidence of the effectiveness of the PA's timely scaffolding and feedback in facilitating learners' (in the PF condition) metacognitive monitoring and regulation during learning.
\end{abstract}

Keywords: self-regulated learning, metacognition, pedagogical agents, co-adaptation, multi-agent systems, learning, product data, process data. 


\section{Objectives and Theoretical Framework}

When learning about complex science topics such as the human circulatory system, research indicates that individuals can gain deep conceptual understanding through effective use of self-regulated learning (SRL). The successful use of cognitive and metacognitive SRL processes involves setting meaningful goals for one's learning, planning a course of action for attaining these goals, deploying a diverse set of effective learning strategies in pursuit of the goals, continuously monitoring one's own understanding of the material and the appropriateness of the current information, and making adaptations to one's goals, strategies, and navigational patterns based on the results of such monitoring processes and resulting judgments [1,2,3,4]. Although learners should attempt to follow these guidelines when attempting difficult topics, exploration of typical learning has demonstrated that few learners, in fact, engage in effective self-regulated learning. Although motivation and affect play a role in determining learners' willingness to self-regulate, we assume a lack of self-regulatory skills is the main obstacle to adequate regulation and, subsequently, deficient learning gains and conceptual understanding [5,6]. Therefore, the current research makes use of pedagogical agents (PAs) to assist learners during interactions with MetaTutor, a multi-agent adaptive hypermedia learning environment that models, scaffolds, and fosters learners' use of cognitive and metacognitive SRL processes during learning about the human circulatory system.

Learners attempting to self-regulate often face limitations in their own metacognitive skills, which, when compounded with lack of domain knowledge, can result in cognitive overload in open-ended learning environments [7,8,9]. One method of relieving the cognitive burden placed on learners in this situation is to provide assistance in the form of adaptive scaffolding. Previous experiments conducted by Azevedo and colleagues [e.g., 10,11] established that adaptive scaffolding provided by a human tutor leads to greater deployment of sophisticated planning processes, metacognitive monitoring processes, and learning strategies as well as larger shifts in mental models of the domain. The purpose of the current work is to determine if adaptive scaffolding provided by PAs within an adaptive, intelligent hypermedia learning environment is also capable of producing the same, or better, learning outcomes and increased use of effective SRL processes.

The current experiment used a mixed-methodology design that combined product and process data to examine the effect of various types of SRL prompting and scaffolding delivered by PAs in an adaptive intelligent hypermedia learning environment. Three learning conditions were used to determine the efficacy of scaffolding SRL through pedagogical agents: 1) prompting with feedback condition (PF), 2) prompting only condition (PO), and 3) no prompting condition (NP). Participants were randomly assigned to one of the three conditions and asked to learn about the human circulatory system using MetaTutor during a two-session experiment. This experiment included the collection of concurrent think-aloud protocols, eye-tracking data, human-agent dialogue, learning outcome measures, log-file data, metacognitive judgments during learning, embedded quizzes, and facial recognition data for affect classification. Due to the complexity of the data analyses, we only report the learning outcomes (i.e., 
learning efficiency) and a few of the log-file variables that are indicative of learners' use of SRL processes.

\section{Method}

\subsection{Participants}

Participants were 69 undergraduate students (75\% females) from a large public university in North America. The mean age of the participants was 23 and their mean GPA was 2.84. All participants were paid $\$ 10$ per hour, up to $\$ 40$ for completion of the 2-day, 4-hour experiment.

\subsection{Materials and MetaTutor}

Materials consisted of several computerized elements. The pretest and posttest each included 25 multiple-choice items each with four foils. Items on the pretest and posttest included text-based items (which could be answered by directly referring one sentence within the content) and inferential items (which required integrating information from at least two sentences within the content). Two equivalent forms of the test were created using a total of 50 items and the forms used for pretest and posttest were counterbalanced across participants.

The learning environment used by all participants, MetaTutor, is an adaptive hypermedia learning environment including 41 pages of text and static diagrams, organized by a table of contents displayed in the left pane of the environment (see Figure 1). The version of MetaTutor used in this experiment includes material related to the human circulatory system. Along with the table of contents, the environment includes a timer indicating time remaining, an SRL palette which learners may use to instantiate an interaction with the pedagogical agent (e.g., indicate that they want to take notes), and an overall learning goal (which was the same for all participants) and sub-goals (which were created by all participants at the beginning of the learning session with the assistance of one of the PAs). Additionally, four distinct pedagogical agents (Gavin, Pam, Mary, and Sam) are displayed in the upper right-hand corner of the environment, which provide varying degrees of prompting and feedback throughout the learning session designed to scaffold students' SRL skills and content understanding.

\subsection{Instructional Conditions}

We designed and tested three versions of the MetaTutor environment. In the Prompt and Feedback (PF) version, participants were prompted by PAs to use specific selfregulatory processes (e.g., metacognitvely monitor their emerging understanding of the topic), and given immediate feedback about their use of those processes. In the Prompt only (PO) version, participants received the same prompts as the ones provided to those in the PF version. However, the agents in the PO version did not 
provide feedback. The timing of the prompts used in both the PF version and the PO version was adaptive to the individual learner and was determined using various factors of learner interaction, including time on page, time on current sub-goal, number of pages visited, relevancy of the current page for the current sub-goal, etc. In the No Prompt (NP) version, participants did not receive prompts or feedback. All three versions (PF, PO, NP) provided an SRL palette, which allowed participants to self-select any SRL processes they wanted to use during the learning session.

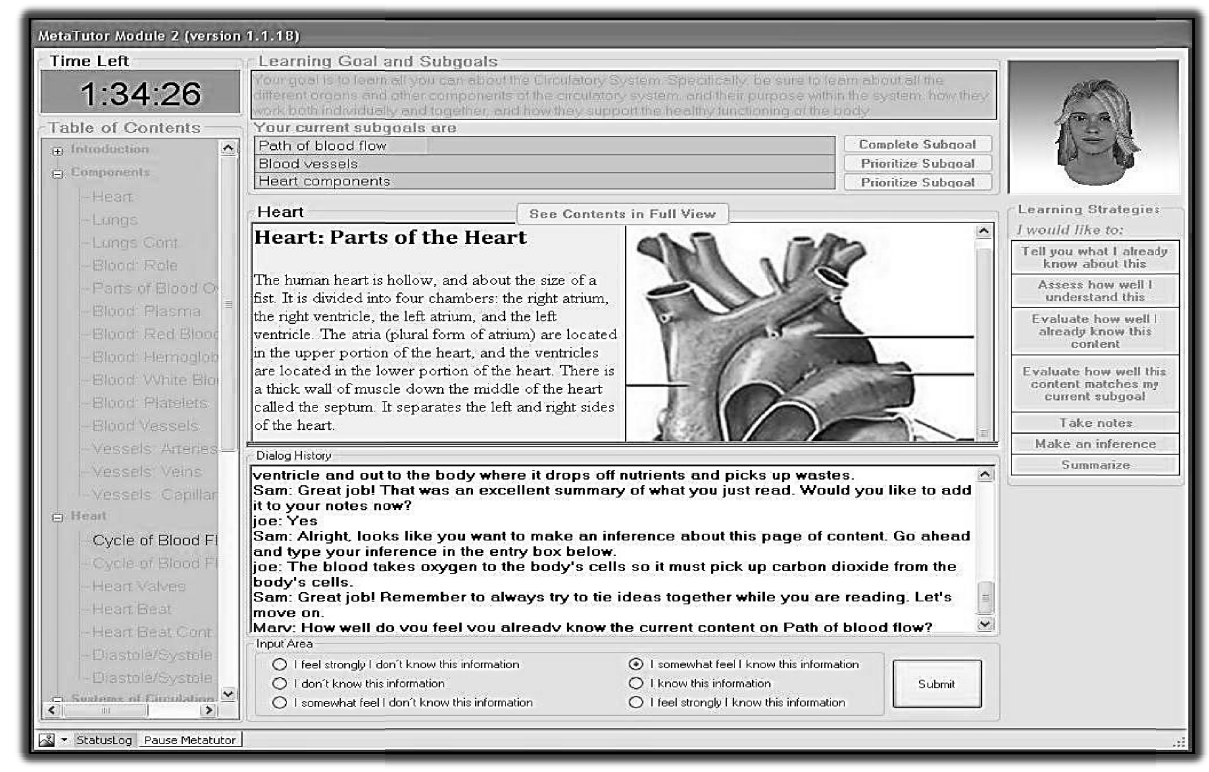

Fig. 1. Screenshot of the MetaTutor Interface

\subsection{Experimental Procedure}

On day one of the experiment, participants completed a demographics questionnaire and the pretest on the human circulatory system. Learners were given up to $20 \mathrm{mi}$ nutes to complete the pretest. On day two, participants engaged in the learning session and completed the posttest on the human circulatory system. Before beginning the learning session, the Tobii T60 eye-tracker was calibrated to each participant individually. All participants were then instructed in the think-aloud procedure and shown a short video demonstrating thinking aloud. Next, each participant was shown another short video explaining and demonstrating the various functionalities of MetaTutor and providing the learners with their overall learning goal (see Figure 1). This introductory video also demonstrated the use of an electronic note-taking feature within the environment and instructed the participants to use the peripheral drawing pad if and when they chose to draw. Following the introductory videos, the learners were given two hours to learn about the human circulatory system using MetaTutor. All participants were provided the opportunity to take a short break ( 5 minutes) during the two 
hours, although not all chose to do so. During the learning session, participant verbalizations and facial expressions were recorded using a Microsoft Lifecam(TM) within the eye-tracker monitor. Immediately after the learning session, participants were given up to 20 minutes to complete the posttest. Finally, all participants were paid and debriefed before leaving the lab.

\section{Results}

In this section we present the learning outcomes (expressed as learning efficiency) and a subset of the log-file data.

Learning Time with the Science Content. Learning time was calculated by summing the amount of time spent viewing the instructional content (i.e., text and diagrams). Interactions with the agents, in which the instructional content was not visible, were not included in learning time. One-way analysis of variance (ANOVA) indicated a significant difference between the groups in learning time, $F(2,66)=$ $40.71, p<.001$. LSD post-hoc analyses indicated that the Control group had a longer total learning time $(M=87.94, S D=12.42)$ when compared to both the PO condition $(M=68.31, S D=11.18)$ and the PF condition $(M=56.84, S D=11.82), p<.001$. Additionally, the PO condition had a significantly longer learning time compared to the PF condition, $p<.01$.

Number of Content Pages Visited. One-way ANOVA also indicated a significant difference between the groups in the mean number of pages visited (out of 41 possi$\left.b^{1}{ }^{1}\right)$ during the learning session, $F(2,66)=22.17, p<.001$. LSD post-hoc analyses revealed that the Control group visited significantly more pages $(M=38.87, S D=$ $3.84)$ than both the PO condition $(M=33.26, S D=8.39 ; \mathrm{p}<.05)$ and the PF condition $(M=23.56, S D=10.07 ; p<.001)$. Additionally, the PO condition visited significantly more pages than the PF condition, $p<.001$.

Amount of Time Spent Reading Pages and Inspecting Diagrams. Results indicated that students did not differ significantly in the amount of time spent on each page (see Table 1). On average, students spent between 60 seconds to 90 seconds on each page $(p>.05)$. By contrast, one-way ANOVA revealed a statistically non- significant difference between groups in the mean time spent viewing individual diagrams within the environment, $F(2,66)=3.02, p=.052$. Given the observed level of marginally significant differences, LSD post-hoc analyses were conducted and revealed that mean diagram view time was greater for the PF condition $(M=1.05 \mathrm{~min}, S D=0.99)$ compared to the Control condition ( $M=0.54 \mathrm{~min}, S D=0.46), \mathrm{p}=.016$. The PO condition did not differ significantly from the remaining two conditions $(M=0.75 \mathrm{~min}$, $S D=0.51$ ).

Number of Sub-Goals Generated during Learning. One-way ANOVA indicated a significant difference between the groups in the number of sub-goals generated during

${ }^{1}$ Subsequent revisits to the same page were not counted in the total. 
the learning session, $F(2,66)=8.74, p<.001$. LSD post-hoc analyses revealed that the PO condition $(M=4.13, S D=1.29)$ and the Control condition $(M=4.70, S D=$ 1.72) both attempted significantly more sub-goals than the PF condition $(M=3.04$, $\mathrm{SD}=0.98), p<.01$. There was not a significant difference between the PO condition and the Control condition. One-way ANOVA indicated a significant difference between the groups in the mean time spent on each individual sub-goal during the learning session, $F(2,66)=10.31, p<.001$. LSD post-hoc analyses revealed that the PF condition $(M=41.39, S D=18.62)$ spent significantly longer on each sub-goal compared to both the PO condition $(M=27.77, S D=9.96)$ and the Control condition $(M$ $=23.30, S D=12.18), p<.01$.

Learning Efficiency ${ }^{2}$. One-way ANOVA on the learning efficiency scores indicated a significant effect of learning condition on learners learning efficiency $(F[2,66]=$ 6.64, $p<.01)$. Post-hoc comparisons revealed that the Prompt and Feedback (PF) condition significantly outperformed the No Prompt (NP) condition $(d=0.84)$. Nonsignificant differences were demonstrated for each of the remaining two comparisons $(p>.05)$. See Table 1 for descriptive statistics.

Table 1. Means (and Standard Deviations) for Various Measures by Condition

\begin{tabular}{|l|c|c|c|}
\hline & $\begin{array}{c}\text { NP Condition } \\
\text { (No Prompt } \\
\text { Condition) } \\
\text { M (SD) }\end{array}$ & $\begin{array}{c}\text { PO Condition } \\
\text { (Prompt } \\
\text { Only) } \\
\text { M (SD) }\end{array}$ & $\begin{array}{c}\text { PF Condition } \\
\text { (Prompt and } \\
\text { Feedback) } \\
\text { M (SD) }\end{array}$ \\
\hline $\begin{array}{l}\text { *Overall Learning Time } \\
\text { (with instructional material only) } \\
\text { (min.) }\end{array}$ & $87.94(12.42)$ & $68.31(11.18)$ & $56.84(11.82)$ \\
\hline *Number of Pages Visited & $38.87(03.84)$ & $33.26(08.39)$ & $23.56(10.07)$ \\
\hline Overall Mean Time on Page (min.) & $1.07(00.66)$ & $0.99(00.50)$ & $1.32(01.06)$ \\
\hline $\begin{array}{l}\text { Overall Mean Time on Diagrams } \\
\text { (min.) }\end{array}$ & $0.54(00.46)$ & $0.75(00.51)$ & $1.05(00.99)$ \\
\hline $\begin{array}{l}\text { *Number of Sub-Goals Set During } \\
\text { Learning Session }\end{array}$ & $4.70(01.72)$ & $4.13(0.1 .29)$ & $3.04(00.98)$ \\
\hline $\begin{array}{l}\text { *Mean Time Spent on Self-Set Sub- } \\
\text { Goal (min.) }\end{array}$ & $23.30(12.18)$ & $27.77(09.96)$ & $41.39(18.60)$ \\
\hline *Learning Efficiency (\%) & $23.10(06.00)$ & $28.90(10.40)$ & $34.30(13.60)$ \\
\hline
\end{tabular}

Note: $* \mathrm{p}<.05$

${ }^{2}$ Each participant received one point for each correct answer selected on the pretest and posttest. From this value, a learning efficiency score was calculated by dividing the raw posttest score by the number of minutes the participant was actually learning (time on task). Time on task was defined as the sum of all of the time spent viewing domain-related content (text and/or diagram). During certain periods of the learning session, the learning content was hidden from view due to interactions with the agent. To account for differential learning time, the time each participant spent viewing the learning content was factored in to the learning efficiency score (Faw \& Waller, 1976; Simons, 1983). 


\section{Discussion}

Current results show that college students' learning about a challenging science topic with hypermedia can be facilitated if they are provided with adaptive prompting and feedback scaffolding designed to regulate their learning. More importantly, we have demonstrated that PAs are effective in facilitating students' SRL processes by providing timely prompting and feedback. Their effectiveness stems from the system's ability to determine optimal times during a learning session (e.g., prompting learners to activate their prior knowledge at the beginning of each generated sub-goal; prompting students to assess whether the current text and diagram are relevant for the current sub-goal). We have demonstrated the effectiveness of prompting and feedback by showing that students in this condition (i.e., PF condition) read less material and navigated through fewer hypermedia pages during the learning task. They also tended to spend more time on each page and spend more time inspecting each diagram presented in MetaTutor. Those in the PF condition also set fewer sub-goals but they spent more time on each sub-goal. Overall, the data support existing theoretical frameworks and models of SRL [e.g., 1,3] related to the use of computers as MetaCognitive tools [1,2]. Subsequent analyses of the verbal protocols, metacognitive judgments, emotions data, and log-file data will allow us to extend current models of SRL and build more sophisticated intelligent multi-agent technology-learning environments designed to detect, trace, model, and foster students' SRL.

Our study contributes to an emerging field that merges educational, cognitive, learning, and computational sciences by addressing issues related to learning about complex science topics with multi-agent environments $[1,5,6,8,9,12]$. Our study also contributes to an emerging body of evidence which illustrates the critical role of SRL in students' learning with hypermedia $[1,2,6,8,11]$, and extends recent research regarding the role of intelligent, adaptive scaffolding in facilitating students' learning with hypermedia [13]. Converging temporally-aligned, multi-level data will allow us to examine the critical role of PAs as external regulatory agents whose scaffolding methods facilitate students' self-regulated learning $[1,8,12]$. Lastly, both our product and process data can be applied to inform the design of intelligent multi-agent hypermedia environments as Metacognitive tools to foster learners' self-regulated learning of challenging science topics by providing adaptive scaffolding $[1,5,6,8,14]$.

\section{Current and Future Directions}

In this paper we presented a few product measures to assess the effectiveness of agents' prompting in supporting learners' SRL processes during learning with MetaTutor. We are currently analyzing huge amounts of data collected from several methods (i.e., eye-tracking, log-file, affect classification, concurrent think-alouds, notes and drawings, learner-agents dialogue, metacognitive judgments, on-line summaries, use of SRL palette). In this section, we present several directions we're currently exploring to enhance our understanding of the various conceptual, theoretical, 
methodological, and analytical issues related to SRL and the potential of multi-agent learning environments.

Measuring SRL with Multi-agent Learning Environments. Multi-agent technology-based learning environments have become popular educational and research tools [12]. Researchers are using them as educational tools to foster learning about complex and challenging topics and domains since embodied pedagogical agents can be programmed to detect, track, model, and foster students' self-regulatory processes, such as planning, metacognitive monitoring, strategy selection and deployment, regulation of affect, motivational beliefs, and reflection [1,9]. In addition, agent-based environments are also being used as research tools to measure the deployment of selfregulatory processes by allowing researchers to collect rich, multi-stream data, including self-report measures of self-regulated learning (SRL), on-line measures of cognitive and metacognitive processes, dialogue moves regarding agent-student interactions, natural language processing of help-seeking behavior, physiological measures of motivation and emotions, emerging patterns of effective problem solving behaviors and strategies, traces of inquiry cycles, etc. In addition, collecting various data streams is critical to enhancing our understanding of when, how, and why students regulate or don't regulate their learning and adapt their regulatory behaviors $[15,16,17]$.

Unique Measurement and Data Analytic Challenges. The current experimental protocol provides a rich source of data through multiple, temporally connected channels. Although our reported analyses relied exclusively on comparisons between experimental groups separately for particular process and outcome variables, the nature of our data is substantially more complex. For example, because SRL processes unfold temporally, we ultimately want to map emotional and or cognitive reactions at one point in time to responses within and across channels at later points in time. Such processes will provide a much more comprehensive picture of the learning process and will allow us to not only identify pre-post performance differences, or simple mean differences across groups, but also to model the intraindividual growth trajectories that underlie learning.

Using MetaTutor to Measure Temporal Dynamics of SRL during Complex Learning. We are synthesizing the results, emphasizing issues and insights that relate to the strengths and weaknesses of collecting, coding, analyzing, and interpreting process data [e.g., see 1]. One issue is the importance of the classification of these processes at various levels of granularity and valence. For example, macro-level (e.g., monitoring process) and micro-level classifications (e.g., monitoring process such as judgment of learning [JOL]) supplemented with valence (i.e., positive or negative [e.g., JOL+]) are key to understanding the multi-level nature of these processes (and inter-related feedback mechanisms) and serve to augment current conceptions and theoretical frameworks of SRL [3]. We are also dealing with the temporal alignment of several data streams (e.g., concurrent think-alouds with eye-tracking data), which are key to understanding the unfolding of the processes in real time and providing evidence of behavioral signatures associated with specific SRL processes. For example, some on-line measures need to be augmented with other measures and methods in 
order to provide converging evidence. The use of log-file data to generate hypotheses regarding fundamental assumptions about SRL (e.g., agency, individual agent's adaptations, and co-adaptations between human and artificial agent during learning). We are also exploring ways in which on-line measures can be converged with other process, product, and self-report data to provide a comprehensive understanding of SRL measurement during learning with multi-agent learning environments.

\section{Co-Regulated Learning between Human and Artificial Pedagogical Agents in the} Context of a Multi-agent Adaptive Hypermedia Environment. Co-adaptation between human and artificial agents is a core issue in the ITS community [see 19]. Contemporary research on multi-agent learning environments has focused on SRL while relatively little effort has been made to use co-regulated learning as a guiding theoretical framework. This oversight needs to be addressed given the complex nature that self-and other-regulatory processes play when human learners and artificial pedagogical agents interact to support learners' internalization of SRL processes [see 19]. For example, learning with a multi-agent hypermedia environment such as MetaTutor involves having a learner interact with four artificial pedagogical agents. Each agent plays different roles including modeling, prompting, and scaffolding SRL processes (e.g., planning, monitoring, and strategy use) and providing feedback regarding the appropriateness and accuracy of learners' use of SRL processes. Accordingly, we are dealing with the challenges and opportunities of our methodological and analytical approaches. One challenge involves determining how our (current study and) research can be re-conceptualized within the framework of co-regulated learning. By doing so, we will extend the human and computerized theoretical models typically used in this research area.

Acknowledgements. The research presented in this paper has been supported by funding from the National Science Foundation (DRL 0633918 and IIS 0841835) awarded to the first author and (DRL 1008282) awarded to the second author.

\section{References}

1. Azevedo, R., Moos, D., Johnson, A., Chauncey, A.: Measuring cognitive and metacognitive regulatory processes used during hypermedia learning: Issues and challenges. Educational Psychologist 45, 210-223 (2010)

2. Azevedo, R., Witherspoon, A., Chauncey, A., Burkett, C., Fike, A.: MetaTutor: A MetaCognitive tool for enhancing self-regulated learning. In: Pirrone, R., Azevedo, R., Biswas, G. (eds.) Proceedings of the AAAI Fall Symposium on Cognitive and Metacognitive Educational Systems, pp. 14-19. Association for the Advancement of Artificial Intelligence (AAAI) Press, Menlo Park (2009)

3. Winne, P.H., Nesbit, J.C.: Supporting self-regulated learning with cognitive tools. In: Hacker, D.J., Dunlosky, J., Graesser, A.C. (eds.) Handbook of Metacognition in Education. Erlbaum, Mahwah (2009)

4. Zimmerman, B., Schunk, D.: Handbook of self-regulation of learning and performance, pp. 102-121. Routledge, New York (2011) 
5. Schwartz, D.L., Chase, C., Chin, D.B., Oppezzo, M., Kwong, H., Okita, S., et al.: Interactive metacognition: Monitoring and regulating a Teachable Agent. In: Hacker, D.J., Dunlosky, J., Graesser, A.C. (eds.) Handbook of Metacognition in Education, pp. 340-358. Routledge, New York (2009)

6. White, B., Frederiksen, J., Collins, A.: The interplay of scientific inquiry and metacognition: More than a marriage of convenience. In: Hacker, D.J., Dunlosky, J., Graesser, A.C. (eds.) Handbook of Metacognition in Education, pp. 175-205. Routledge, New York (2009)

7. Azevedo, R., Cromley, J.G., Moos, D.C., Greene, J.A., Winters, F.I.: Adaptive content and process scaffolding: A key to facilitating students' self-regulated learning with hypermedia. Psychological Testing and Assessment Modeling 53, 106-140 (2011)

8. Leelawong, K., Biswas, G.: Designing learning by teaching agents: The Betty's Brain System. International Journal of Artificial Intelligence in Education 18, 181-208 (2008)

9. Robison, J., Rowe, J., McQuiggan, S., Lester, J.: Predicting User Psychological Characteristics from Interactions with Empathetic Virtual Agents. In: Ruttkay, Z., Kipp, M., Nijholt, A., Vilhjálmsson, H.H. (eds.) IVA 2009. LNCS, vol. 5773, pp. 330-336. Springer, Heidelberg (2009)

10. Azevedo, R., Johnson, A., Chauncey, A., Graesser, A.: Use of hypermedia to convey and assess self-regulated learning. In: Zimmerman, B., Schunk, D. (eds.) Handbook of SelfRegulation of Learning and Performance, pp. 102-121. Routledge, New York (2011)

11. Azevedo, R., Witherspoon, A.M.: Self-regulated use of hypermedia. In: Hacker, D.J., Dunlosky, J., Graesser, A.C. (eds.) Handbook of Metacognition in Education, pp. 319-339. Routledge, New York (2009)

12. Graesser, A.C., McNamara, D.S.: Self-regulated learning in learning environments with pedagogical agents that interact in natural language. Educational Psychologist 45, 234-244 (2010)

13. Vanlehn, K.: The relative effectiveness of human tutoring, intelligent tutoring systems and other tutoring systems. Educational Psychologist 46(4), 197-221 (2011)

14. Aleven, V., Roll, I., McLaren, B.M., Koedinger, K.R.: Automated, unobtrusive, action-byaction assessment of self-regulation during learning with an intelligent tutoring system. Educational Psychologist 45, 224-233 (2010)

15. Calvo, R., D'Mello, S.K. (eds.): New perspectives on affect and learning technologies. Springer, New York (2011)

16. Azevedo, R., Aleven, V. (eds.): International handbook of metacognition and learning technologies. Springer, Amsterdam (in press)

17. D’Mello, S.K., Graesser, A.C.: Dynamics of affective states during complex learning. Learning and Instruction 22, 145-157 (2012)

18. Kinnebrew, J., Biswas, G., Sulcer, B., Taylor, R.: Investigating self-regulated learning in Teachable Agent environments. In: Azevedo, R., Aleven, V. (eds.) International Handbook of Metacognition and Learning Technologies. Springer, Berlin (in press)

19. Hadwin, A.F., Järvelä, S., Miller, M.: Self-regulated, co-regulated, and socially-shared regulation of learning. In: Zimmerman, B.J., Schunk, D.H. (eds.) Handbook of SelfRegulation of Learning and Performance, pp. 65-84. Routledge, New York (2011) 